\title{
Influence of the crystal orientation of substrate on low temperature synthesis of silicon nanowires from $\mathrm{Si}_{2} \mathrm{H}_{6}$
}

\author{
Saeed Akhtar ${ }^{\text {a,* }}$, A. Tanaka ${ }^{\text {a }}$, K. Usami ${ }^{\text {a }}$, Y. Tsuchiya ${ }^{\text {a }}$, S. Oda ${ }^{\text {a,b }}$ \\ a Quantum Nanoelectronics Research Center, and Tokyo Institute of Technology, 2-12-1 O-okayama, Meguro-ku, Tokyo 152-8552, Japan \\ b Solution Oriented Research for Science and Technology, JST, Kawaguchi, Saitama 332-0012, Japan
}

\section{A R T I C L E I N F O}

Available online 27 August 2008

\section{Keywords:}

Si nanowires

Synthesis

Diffusion

Diameter distribution

Au Catalyst

Eutectic temperature

Low pressure chemical vapor deposition

\begin{abstract}
A B S T R A C T
SiNW synthesis by vapor-liquid-solid (VLS) growth mechanism has been reported by many authors but a deep understanding of the key factors affecting the nanowire growth is still lacking. Here, we report a thorough investigations of the SiNW growth by employing silicon substrates with different crystal orientations and surface preparation using Au thin film as a catalyst. We observed that the activity of the Au particles strongly depends on the crystal orientation and the substrate surface preparation significantly affects the properties of SiNWs. We found that the density and growth rate of nanowires is different in a temperature dependent growth on $\mathrm{Si}(100)$ from $\mathrm{Si}(111)$. We found that the density and growth rate of nanowires is different at different crystal orientations of the substrates. We have grown nearly uniform diameter and micrometer long SiNWs by using $\mathrm{Si}_{2} \mathrm{H}_{6}$ as a source gas in low pressure chemical vapor deposition around eutectic temperature. The length of the SiNWs can be controlled mainly by varying the growth time.
\end{abstract}

(c) 2008 Elsevier B.V. All rights reserved.

\section{Introduction}

Recently silicon nanowires (SiNWs) have been intensively studied due to the proposed, partly fuelled by the proposed applications in future nanoelectronics and photonic devices $[1,2]$. The Si one dimensional nano structures could be used as a critical component for nanoelectronic devices acting as a functional unit as well as interconnects. The quantum confinement effects on electrical and optical properties of the wires are strongly size dependent making the SiNW growth with small and finite diameters a prerequisite for exploiting such unique properties for device fabrication purposes. The modifications of the diameter of SiNWs by using different ambient gases [3], ambient pressure [4], and uniform diameter catalyst colloids have already been reported $[5,6]$. In most of the cases SiNWs were grown by the vapor-liquid-solid (VLS) mechanism using different approaches. Even though, SiNW growth using VLS mechanism has been investigated over the years but information regarding controlled synthesis is still lacking. Here we report the influence of $\mathrm{Si}_{2} \mathrm{H}_{6}$ precursor gas and crystal orientation of the substrate and surface preparation on low temperature synthesis of SiNWs by using Au as a catalyst in VLS low pressure

\footnotetext{
* Corresponding author. Tel./fax: +8135734 2542.

E-mail address: saeed@neo.pe.titech.ac.jp (S. Akhtar).
}

chemical vapor deposition. We have grown bulk quantities of SiNWs at low temperature, around the bulk Si-Au eutectic temperature, indicating the process suitability for mass production and large scale device fabrication.

In this paper, we discuss the density and the growth rate of the SiNW dependence on the crystal orientation and surface conditions of the substrate. Mainly we have investigated the SiNW synthesis on $\mathrm{Si}(111)$ and $\mathrm{Si}(100)$ substrates by using Au thin film as a catalyst. We found that the activity of the Au catalyst particles strongly depends on the crystal face of the substrate used. The size distribution of initial Si-Au alloy droplet depends on growth temperature, which consequently defines the diameter distribution of SiNWs. After that we will demonstrate under same growth conditions density, growth rate, and morphology of nanowires is different at different crystal faces of the substrates. We observed that the crystal orientation of the substrate has a strong influence on the SiNW growth process.

We have grown SiNWs under low temperature and low-pressure conditions by using Au as a catalyst. There are many advantages of using $\mathrm{Au}$ as a seed to direct the nanowire growth. The bulk eutectic temperature of $\mathrm{Si}-\mathrm{Au}$ system is about $363^{\circ} \mathrm{C}$ and also it does not form silicide. Recently SiNWs have been grown at below eutectic temperature from $\mathrm{Si}-\mathrm{Cu}$ system [7]. Also the germanium nanowire synthesis from $\mathrm{Ge}-\mathrm{Au}$ system below eutectic temperature has been reported [8]. Our nanowire growth process from $\mathrm{Si}-\mathrm{Au}$ system is simple and gives faster growth rate, high density, and fixed diameter micrometer long nanowires. 


\section{Experimental details}

In our experiments, first $\mathrm{Si}(100)$ and $\mathrm{Si}(111)$ substrates were cleaned in a standard process and then treated with HF just before loading into the evaporation chamber through air. The substrates were dipped into $1.5 \%$ HF for about three mins presumably etch away the top Si layers. The Au thin film of $1 \mathrm{~nm}$ was deposited by electron beam evaporation at room temperature. Thickness of the Au film was measured by using quartz crystal monitor. Afterwards, the samples were transferred into low pressure chemical vapor deposition chamber and typically exposed to a flow of $1 \mathrm{sccm}$ of $\mathrm{Si}_{2} \mathrm{H}_{6}$ and 49 sccm of $\mathrm{H}_{2}$ at a pressure of 3 Torr and $350{ }^{\circ} \mathrm{C}$. In our experiments, growth temperature is measured at the outer wall of the horizontal chamber [9]. SiNW growth was carried out for 16 to 60 minutes.

\section{Results and discussions}

Scanning electron microcopy analysis revealed that electron beam evaporated Au thin film forms droplets like morphology on silicon substrates (see Fig. 1a). The average size distribution of Au droplets was found on the order of $12 \pm 5 \mathrm{~nm}$, which is shown in a bar graph in Fig. 1b. However, at elevated temperatures the activity of the $\mathrm{Au}$ particles have strong dependence on the crystal orientation of the substrates.

The activity of the Au particles at higher temperatures affects the properties of the SiNWs. It was observe that large quantity of $\mathrm{Au}$ droplets aggregate to $\mathrm{Si}(100)$ substrate and do not take part in the growth process of SiNWs giving rise to very low yield. On the other hand, we found that all Au droplets take part in silicon nanowire growth process on $\mathrm{Si}(111)$ substrate resulting in about $75 \%$ higher nanowire yield than on $\mathrm{Si}(100)$ substrate. This indicates that the crystal orientation of the substrate plays very important role in the

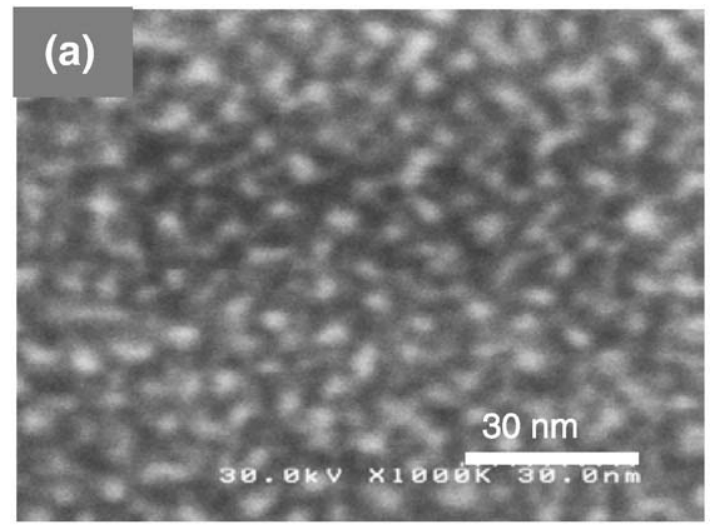

(b)

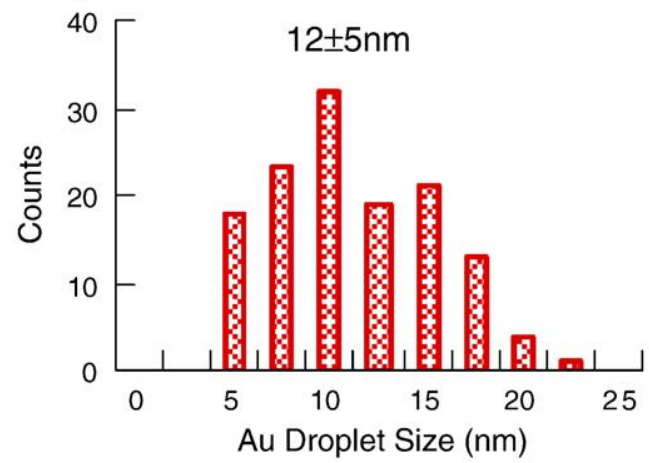

Fig. 1. (a) SEM image of e-beam evaporated $1 \mathrm{~nm}$ Au droplet film thickness measured with the quartz crystal monitor. (b) Size distribution of Au droplet. $12 \pm 5 \mathrm{~nm}$.

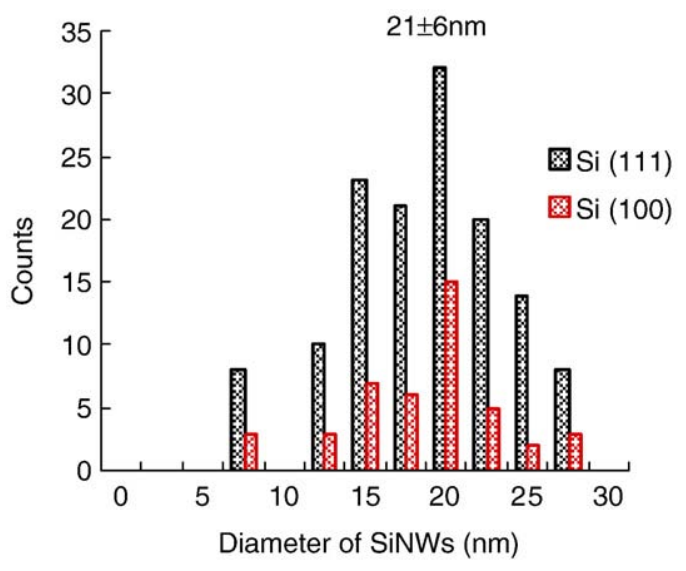

Fig. 2. Size distribution of $\mathrm{Si}$ nanowires grown on $\mathrm{Si}(111)$ and $\mathrm{Si}(100)$ substrate for $180 \times 180 \mathrm{~nm}^{2}$ area.

growth process of SiNWs. The surface conditions of the substrate are assumed to affect the growth as well. A glaring dependence of the activity of the Au particles on crystal orientation of the substrate was observed; where all Au particles nucleate SiNWs on $\mathrm{Si}(111)$ resulting in high density nanowire synthesis. The variations in density and the growth rate depending on different substrates are typically observed in our experiments. We found that the nanowires grow at much faster growth rate on $\mathrm{Si}(111)$ than on $\mathrm{Si}(100)$ substrate. The average diameter distribution of SiNWs grown from $1 \mathrm{~nm}$ Au film on both types of substrates was found about $21 \pm 6 \mathrm{~nm}$. Comparative detail of SiNWs density and diameter distribution on $\mathrm{Si}(111)$ and $\mathrm{Si}(100)$ substrate has given in Fig. 2.

Bulk quantities of SiNWs were grown on $\mathrm{Si}(111)$ substrate by using VLS low-pressure chemical vapor deposition mechanism (Fig. 3). SiNWs can controllably be grown from one to tens of microns in length by mainly varying the growth time. SiNW density on Si(111) substrate was found about $27 \pm 3$ wires $/ \mu \mathrm{m}^{2}$ with average $27 \mathrm{~nm}$ inter wire spacing. It was found that $\mathrm{Si}(111)$ substrate was responsible for high density, and fast growth rate of SiNWs. Also SiNWs grown on $\mathrm{Si}(111)$ found smooth, clean, and micrometer long showing suitability for applications in nano device fabrication or as interconnects.

High density uniform diameter silicon nanowires were synthesized on $\mathrm{Si}(111)$ in contrast to the $\mathrm{Si}(100)$ substrate. It is well known that the coarsening of the Au particles on silicon substrate during the $\mathrm{Au}-\mathrm{Si}$ eutectic droplet formation process determines the diameter SiNWs were synthesized [10]. In fact mobility and diffusion of Au particles on silicon substrate has a profound effect on various properties of the SiNWs. We observed that Si-Au eutectic alloy

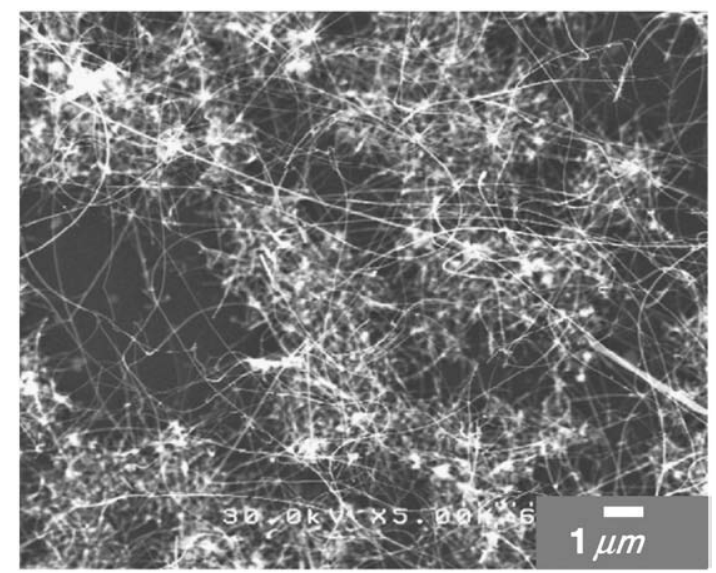

Fig. 3. SEM image of high density silicon nanowires grown on $\mathrm{Si}(111)$ substrate with average diameter distribution of $21 \pm 6 \mathrm{~nm}$. 

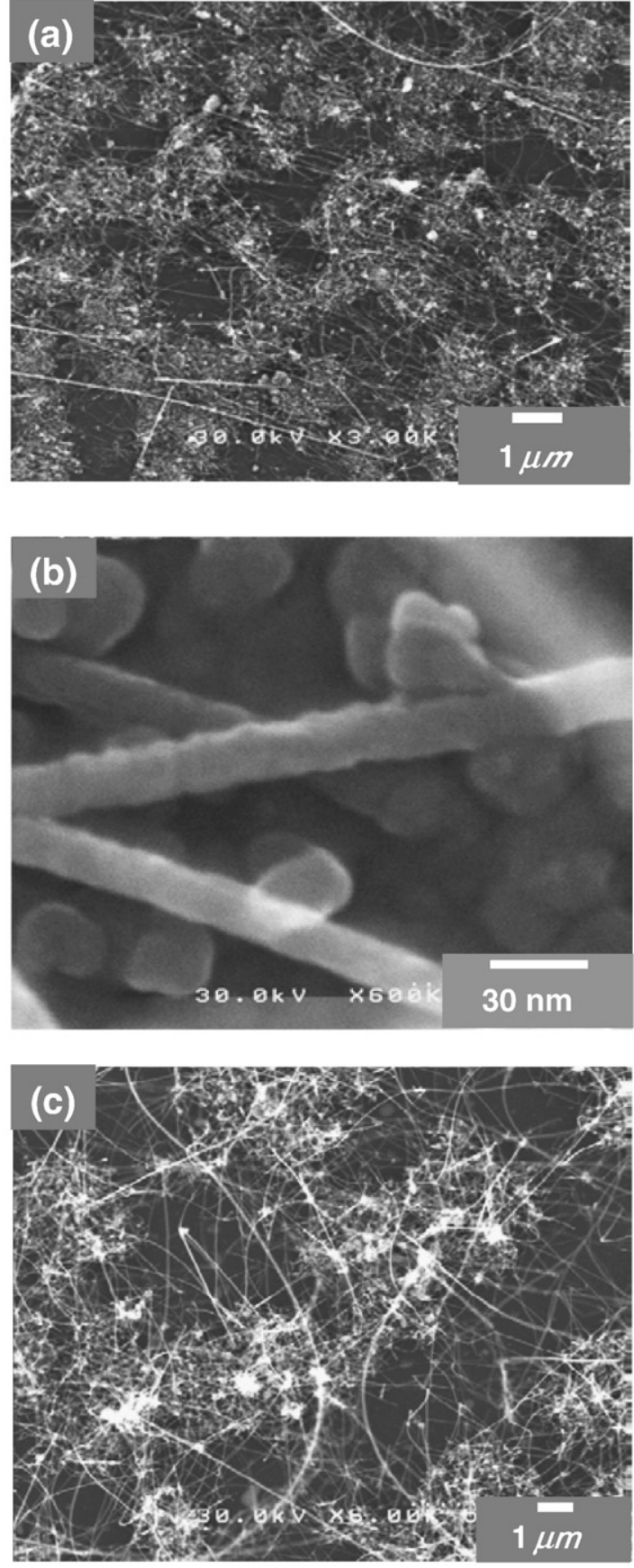

Fig. 4. (a) SEM image of high density silicon nanowires grown from $1 \mathrm{~nm}$ Au thin film deposited on $\mathrm{Si}(111)$ substrate at $350{ }^{\circ} \mathrm{C}$, (b) high resolution SEM image showing $\mathrm{Au}$ particles aggregate to $\mathrm{Si}(100)$ substrate during nanowire growth process and do not nucleate any nanowire, while (c) SEM image of uniform diameter silicon nanowires shows the overall morphology of the silicon nanowires grown on $\mathrm{Si}(111)$.

formation process and consequently the nanowires nucleation process depends on the crystal orientation of the substrate. The SiNWs grown on $\mathrm{Si}(111)$ and $\mathrm{Si}(100)$ substrates provide different morphology. We found that SiNWs grown on $\mathrm{Si}(111)$ gives full coverage of the substrate and very high yield but on $\mathrm{Si}(100)$ substrate silicon atoms form bigger molecules around the Au particles and catalyst particles do not nucleate nanowires (see Fig. 4a and 4b). It is still desired to investigate how the crystal orientation of the substrate affects the nanowire synthesis. We need to investigate further what is the difference in behavior of the Au particles on different crystal faces of the substrates during VLS nucleation process? We grow SiNWs at low temperature where the migration of the Au particles on silicon substrate is negligible and results in high density SiNWs on $\mathrm{Si}(111)$ substrate. Although at low temperature growth process the migration of the $\mathrm{Au}$ particles on $\mathrm{Si}(100)$ is negligible but most of the catalyst particles do not nucleate any nanowires and aggregate to the substrate surface. It is low temperature growth process which results at high density nanowire synthesis. Fig. 4c is the SEM image of high density SiNWs grown at $350{ }^{\circ} \mathrm{C}$ which gives information about over all morphology of the nanowires. The high density and fixed diameter nanowire growth is attributed to the crystal orientation and surface preparation of the substrate and the low temperature growth process.

\section{Conclusion}

In conclusion, we have shown that the activity of the Au particles is different on different crystal orientation of the substrate which considerably affects the density and growth rate of the SiNWs. We found that the $\mathrm{Si}(111)$ substrate gives high nanowire yield as compared to the $\mathrm{Si}(100)$ substrate. The difference in eutectic alloying and nucleation processes on $\mathrm{Si}(111)$ and $\mathrm{Si}(100)$ is supposed to be different depending on the specific substrate surface conditions. It is believed that the low temperature and low-pressure chemical vapor deposition stops the migration of Au particles and gives high density nanowires [10] but we found that the crystal orientation and surface condition another factor which affects the density as well as the growth rate of the nanowires. These results provide deep understanding of the $\mathrm{Si}-\mathrm{Au}$ system dependence on crystal orientation of the substrate in low temperature SiNW synthesis via VLS growth mechanism.

\section{References}

[1] J. Goldberger, A.I. Hochbaum, R. Fan, P. Yang, Nano Lett. 6 (2006) 973.

[2] Y. Huang, X. Duan, C.M. Lieber, Small 1 (2005) 142.

[3] Y.F. Zhang, Y.H. Tang, H.Y. Peng, N. Wang, C.S. Lee, I. Bello, S.T. Lee, Appl. Phys. Lett. 75 (1999) 1842

[4] H.Z. Zhang, D.P. Yu, Y. Ding, Z.G. Bai, Q.L. Hang, S.Q. Feng, App. Phys. Lett. 73 (2001) 3396.

[5] Y. Cui, L.J. Lauhon, M.S. Gudiksen, J. Wang, C.M. Lieber, Appl. Phys. Lets. 78 (2001) 2214.

[6] A.I. Hochbaum, R. Fan, R. He, P. Yang, Nano Lett. 5 (2005) 460.

[7] Y. Yao, S. Fan, Materials Letters 61 (2007) 177.

[8] S. Kodambaka, J. Tersoff, M.C. Reuter, F.M. Ross, Science 316 (2007) 729.

[9] S. Akhtar, K. Usami, Y. Tsuchiya, H. Mizuta, S. Oda, Appl. Phys. Express 1 (2008) 014003.

[10] J.B. Hannon, S. Kodambaka, F.M. Ross, R.M. Tromp, Nature 440 (2006) 69. 